Tôhoku Math. Journ.

39 (1987), 1-15.

\title{
RANDOM FOURIER-STIELTJES SERIES ASSOCIATED WITH STABLE PROCESS
}

\author{
Chanchala Nayak, Swadheenananda Pattanayak, \\ aNd Mahendra Nath Mishra
}

(Received January 7, 1986)

1. Introduction. Let $X(t, w), t \in R$, be a continuous stochastic process with independent increments and $f$ be a continuous function in $[a, b]$. Then the stochastic integral

$$
\int_{a}^{b} f(t) d X(t, w)
$$

is defined in the sense of convergence in probability and is a random variable (cf. Lukacs [4, p. 148]). Hence in particular

$$
A_{n}(w)=\int_{0}^{1} e^{-2 \pi n i t} d X(t, w)
$$

exists for an orthonormal set $e^{2 \pi n i t}$ and is the Fourier-Stieltjes coefficient of $X(t, w)$. The series

$$
\sum_{-\infty}^{\infty} A_{n}(w) e^{2 \pi n i y}
$$

is a Fourier-Stieltjes expansion of $X(y, w)$. The convergence and continuity of the series

$$
\sum_{-\infty}^{\infty} \frac{A_{n}(w)}{n} e^{2 \pi n i y}
$$

was studied by Samal [7]. He has shown that the series (1) converges in distribution and the sum is weakly continuous in probability (see Definition $D_{1}$ in $\S 2$ ). Mishra, Nayak and Pattanayak [6] have shown that under the condition $\sum_{-\infty}^{\infty}\left|a_{n}\right|^{2}<\infty$ the weighted Random Fourier-Stieltjes series (RFS, for short)

$$
\sum_{-\infty}^{\infty} a_{n} A_{n}(w) e^{2 \pi n i y}
$$

converges in probability and is weakly continuous in probability. They have also shown that under the stronger condition $\sum_{-\infty}^{\infty}\left|n a_{n}\right|^{2}<\infty$ the same series converges almost surely at every $y$ and the sum function is strongly continuous in probability (see Definition $D_{3}$ in $\S 2$ ). 
Attention had not been paid to finding the sum to which the RFS series converges. Here our motivation is to find the sum to which the RFS series (2) converges. We discuss in detail the mode of convergence of the said series extending our work in a different direction. In Theorem 1 , considering a symmetric stable process of index $\alpha, 1 \leqq \alpha \leqq 2$, we have defined a new type of stochastic integral for a class of functions in $L^{p}[a, b], p \geqq 1$. In the remaining four theorems we have studied the convergence of the series (2) to a stochastic integral

$$
\int_{0}^{1} f(t-t) d X(t, w) \text {, }
$$

where $f$ is the unique function in $L^{p}[0,1], p \geqq 1$, with the Fourier coefficients $a_{n}$. The mode of convergence depends on the value of $\alpha$. In Theorem 2 we have considered the case $1<\alpha<2$, and have shown that the series (2) converges in probability to the stochastic integral (3) and is weakly continuous in probability. In Theorem 3 , by imposing stronger condition like $\sum_{-\infty}^{\infty}\left|n a_{n}\right|^{2}<\infty$ on the weights $a_{n}$, the sum function has been shown to be differentiable in probability. In Theorem 4, the case $\alpha=2$ is discussed where it is proved that the RFS series converges almost surely to the stochastic integral (3) and the sum function is continuous in quadratic mean, which can be improved to be almost surely continuous function under some stronger condition on weights $a_{n}$. In Theorem 5 , the case $\alpha=1$ is discussed and it is shown that the series (2) though not convergent is convergent $(C, 1)$ in probability (see Definition $D_{5}$ in $\S 2$ ) to the stochastic integral (3). Of course, when $|f| \log ^{+}|f|$ is integrable, the series (2) is convergent as discussed in Corollary to Theorem 5. When $0<\alpha<1$, imposition of a different set of restrictions on weights $a_{n}$ confirms the convergence in probability of the series (2) to the stochastic integral (3) as discussed in Theorem 6 .

\section{Definitions.}

Definition $\mathrm{D}_{1}$. A random function $f(t, w)$ is said to be weakly continuous in probability at $t=t_{0}$ if for all $\delta>0$,

$$
\lim _{h \rightarrow 0} P\left(\left|f\left(t_{0}+h, w\right)-f\left(t_{0}, w\right)\right|>\delta\right)=0 .
$$

A function $f(t, w)$ is weakly continuous in probability in a closed interval $[a, b]$ if it is weakly continuous at every $t_{0} \in[a, b]$.

Definition $D_{2}$. The class of functions $f$ satisfying $\int_{a}^{b}|f(t)|^{p} d t<\infty$ is denoted by $L^{p}[a, b]$. 
Definition $\mathrm{D}_{3}$. A random function $f(t, w)$ is said to be strongly continuous in probability if for all $\delta>0$,

$$
\lim _{n \rightarrow \infty} P\left(\sup _{1 \leqq k \leqq n}\left|f\left(a+\frac{k(b-a)}{n}, w\right)-f\left(a+\frac{(k-1)(b-a)}{n}, w\right)\right|>\delta\right)=0 .
$$

Definition $\mathrm{D}_{4}$. A random function $f(t, w)$ is said to be differentiable in probability at $t=t_{0}$ if there exists a random function $g(t, w)$ such that for all $\delta>0$,

$$
\lim _{h \rightarrow 0} P\left(\left|\frac{f\left(t_{0}+h, w\right)-f\left(t_{0}, w\right)}{h}-g\left(t_{0}, w\right)\right|>\delta\right)=0 .
$$

Definition $D_{5}$. A sequence of random variables $X_{n}$ converges in $(C, 1)$ probability to a random variable $X$ if

$$
\lim _{n \rightarrow \infty} P\left(\left|Y_{n}-X\right| \geqq \varepsilon\right)=0, \text { for all } \varepsilon>0,
$$

where

$$
Y_{n}=\frac{X_{0}+X_{1}+\cdots+X_{n-1}}{n}
$$

\section{Results.}

THEOREM 1. If $X(t, w)$ is a symmetric stable process of index $\alpha$, $1 \leqq \alpha \leqq 2$, then for $f \in L^{p}[a, b], p \geqq 1$

$$
\int_{a}^{b} f(t) d X(t, w)
$$

can be defined in the sense of convergence in probability.

REMARK. As far as we know in all literature hitherto the stochastic integral $\int_{a}^{b} f(t) d X(t, w)$ is defined either for $f \in L^{2}[a, b]$ or $f$, continuous in $[a, b]$. In case $f$ is an $L^{2}$ function the integral exists in the sense of convergence in quadratic mean and when $f$ is a continuous function and $X(t, w)$ is a continuous homogeneous process with independent increment, the stochastic integral exists in the sense of convergence in probability. But the above theorem proves the existence of a stochastic integral for a larger class of functions in $L^{p}, p \geqq 1$.

For the proof of the above theorem we require the following lemma due to Samal and Mishra [8].

LEMMA 1. Let $f(t)$ be any continuous function with continuous derivative in $[a, b]$ and $X(t, w)$ be a symmetric stable process with index $\alpha, 0<\alpha \leqq 2$. Then for all $\delta>0$, 


$$
P\left(\left|\int_{a}^{b} f(t) d X(t, w)\right|>\delta\right) \leqq \frac{C \cdot 2^{\alpha+1}}{(\alpha+1) \delta^{\alpha}} \int_{a}^{b}|f(t)|^{\alpha} d t
$$

where $C$ is a positive constant.

Proof of Theorem 1. It is well known (cf. Zygmund $[9$, p. 265]) that for $f \in L^{p}[a, b], p \geqq 1$, we can get a sequence $f_{n}$ of continuous functions with continuous derivatives in $[a, b]$ such that

$$
\lim _{m \rightarrow \infty} \int_{a}^{b}\left|f_{m}(t)-f(t)\right|^{p} d t=0 .
$$

Thus we get

$$
\lim _{m, n \rightarrow \infty} \int_{a}^{b}\left|f_{m}(t)-f_{n}(t)\right|^{p} d t=0 .
$$

So by application of Lemma 1, we get

$$
P\left(\left|\int_{a}^{b} f_{n}(t) d X(t, w)-\int_{a}^{b} f_{m}(t) d X(t, w)\right|>\delta\right) \leqq \frac{C \cdot 2^{\alpha+1}}{(\alpha+1) \delta^{\alpha}} \int_{a}^{b}\left|f_{n}(t)-f_{m}(t)\right|^{\alpha} d t .
$$

Since $1 \leqq \alpha \leqq 2$ by (5), the above right hand side integral approaches 0 , as $m, n \rightarrow \infty$ and thus the stochastic integral

$$
\int_{a}^{b} f_{m}(t) d X(t, w)
$$

converges in probability. Hence (cf. Kawata [3, p. 479]) there exists a random variable $Y$ such that

$$
\lim _{m \rightarrow \infty} P\left(\left|\int_{a}^{b} f_{m}(t) d X(t, w)-Y\right|>\delta\right)=0 .
$$

It is easy to see that this is independent of the choice of sequence $f_{m}$. Suppose there is a sequence $g_{m}$ such that

$$
\lim _{m \rightarrow \infty} \int_{a}^{b}\left|g_{m}(t)-f(t)\right|^{p} d t=0, \quad p \geqq 1 .
$$

Then we easily get

$$
\lim _{m \rightarrow \infty} \int_{a}^{b}\left|f_{m}(t)-g_{m}(t)\right|^{p} d t=0 .
$$

By Lemma 1 and statement (6), we get

$$
\lim _{m \rightarrow \infty} P\left(\left|\int_{a}^{b} f_{m}(t) d X(t, w)-\int_{a}^{b} g_{m}(t) d X(t, w)\right|>\delta\right)=0 .
$$

We define the random variable $Y$ to be the stochastic integral of $L^{p}$, $p \geqq 1$ function. 
In the next theorem we have described how to approximate the stochastic integral of an $L^{p}$ function to a trigonometric polynomial with random coefficients. For this we require the extension of Lemma 1 for an $L^{p}$ function.

LEMMA 2. Let $f(t)$ be any function in $L^{p}[a, b], p \geqq 1$, and $X(t, w)$ be a symmetric stable process of index $\alpha, 1 \leqq \alpha \leqq 2$. Then for all $\delta>0$,

$$
P\left(\left|\int_{a}^{b} f(t) d X(t, w)\right|>\delta\right) \leqq \frac{C \cdot 2^{\alpha+1}}{(\alpha+1) \delta^{\prime \alpha}} \int_{a}^{b}|f(t)|^{\alpha} d t
$$

where $\delta^{\prime}<\delta$ and $C$ is a positive constant.

Proof of Lemma 2. For $f \in L^{p}[a, b], p \geqq 1$, we have a sequence of continuous functions $f_{m}$ with continuous derivatives, satisfying (5). Now for each $\varepsilon>0$,

$$
\begin{aligned}
P\left(\left|\int_{a}^{b} f(t) d X(t, w)\right|>\delta\right)= & P\left(\left|\int_{a}^{b}\left(f(t)-f_{m}(t)+f_{m}(t)\right) d X(t, w)\right|>\delta\right) \\
\leqq & P\left(\left|\int_{a}^{b}\left(f(t)-f_{m}(t)\right) d X(t, w)\right|>\varepsilon\right) \\
& +P\left(\left|\int_{a}^{b} f_{m}(t) d X(t, w)\right|>\delta-\varepsilon\right) .
\end{aligned}
$$

But by Theorem 1,

$$
\lim _{m \rightarrow \infty} P\left(\left|\int_{a}^{b}\left(f(t)-f_{m}(t)\right) d X(t, w)\right|>\varepsilon\right)=0
$$

and by Lemma 1

$$
\begin{aligned}
& \lim _{m \rightarrow \infty} P\left(\left|\int_{a}^{b} f_{m}(t) d X(t, w)\right|>\delta-\varepsilon\right) \\
& \quad \leqq \frac{C \cdot 2^{\alpha+1}}{(\alpha+1)(\delta-\varepsilon)^{\alpha}} \lim _{m \rightarrow \infty} \int_{a}^{b}\left|f_{m}(t)\right|^{\alpha} d t=\frac{C \cdot 2^{\alpha+1}}{(\alpha+1) \delta^{\prime \alpha}} \int_{a}^{b}|f(t)|^{\alpha} d t
\end{aligned}
$$

(by Minkowski's inequality).

The above result with (7) proves the lemma.

THEOREM 2. Let $X(t, w)$ be a symmetric stable process of index $\alpha$, $1<\alpha<2$, with period one,

$$
A_{n}(w)=\int_{0}^{1} e^{-2 \pi n i t} d X(t, w), \quad n \in z
$$

Then

(a) the RFS series

$$
\sum_{-\infty}^{\infty} a_{n} A_{n}(w) e^{2 \pi n i y}
$$


converges in probability to the stochastic integral

$$
\int_{0}^{1} f(y-t) d X(t, w),
$$

for some $f \in L^{p}(p \geqq 1)$, such that $a_{n}$ is the Fourier coefficient of $f$ and

(b) the sum function is weakly continuous in probability.

REMARK. Theorem 2 is a generalization of Samal and Mishra [8] for $a_{n}=1 / n$ which is the Fourier coefficient of the function $\pi / 2-\pi t$. By restricting the process to a stable one of index $1<\alpha<2$, the convergence in distribution is improved to convergence in probability. Since

$$
f \in L^{2} \Longleftarrow \sum_{-\infty}^{\infty}\left|a_{n}\right|^{2}<\infty
$$

it follows that our result also includes the result of Mishra, Nayak and Pattanayak [6].

Proof of TheOREM 2. Let

$$
S_{n}(y, w)=\sum_{-n}^{n} a_{k} A_{k}(w) e^{2 \pi k i y}
$$

and

$$
f_{n}(t)=\sum_{-n}^{n} a_{k} e^{2 \pi k i t}
$$

Thus

$$
S_{n}(y, w)=\sum_{-n}^{n} \int_{0}^{1} a_{k} e^{2 \pi k i(y-t)} d X(t, w)=\int_{0}^{1} f_{n}(y-t) d X(t, w)
$$

Now

$$
\begin{aligned}
& P\left(\left|\int_{0}^{1} f(y-t) d X(t, w)-S_{n}(y, w)\right|>\delta\right) \\
& \quad=P\left(\left|\int_{0}^{1}\left(f(y-t)-f_{n}(y-t)\right) d X(t, w)\right|>\delta\right) \\
& \quad \leqq \frac{C \cdot 2^{\alpha+1}}{(\alpha+1) \delta^{\prime \alpha}} \int_{0}^{1}\left|f(y-t)-f_{n}(y-t)\right|^{\alpha} d t \quad \text { (by Lemma 2), }
\end{aligned}
$$

where $\delta^{\prime}<\delta$. But we know (cf. Zygmund [9, p. 266]) for $f \in L^{p}, p \geqq 1$, $\alpha>1$

$$
\lim _{n \rightarrow \infty} \int_{0}^{1}\left|f(y-t)-f_{n}(y-t)\right|^{\alpha} d t=0
$$

Hence

$$
\lim _{n \rightarrow \infty} P\left(\left|\int_{0}^{1} f(y-t) d X(t, w)-S_{n}(y, w)\right|>\delta\right)=0, \text { for all } \delta>0 .
$$


Thus the series (9) converges in probability to the stochastic integral (10).

For the second part of the theorem it is enough to look at the inequality

$$
\begin{aligned}
& P\left(\left|\int_{0}^{1} f(x-t) d X(t, w)-\int_{0}^{1} f(y-t) d X(t, w)\right|>\delta\right) \\
& \quad \leqq \frac{C \cdot 2^{\alpha+1}}{(\alpha+1) \delta^{\prime \alpha}} \int_{0}^{1}|f(x-t)-f(y-t)|^{\alpha} d t,<\delta, \quad(\text { by Lemma } 2) .
\end{aligned}
$$

But for $f \in L^{p}, p \geqq 1$, we know (cf. Zygmund [9, p. 37])

$$
\lim _{x \rightarrow y}\left(\int_{0}^{1}|f(x-t)-f(y-t)|^{p} d t\right)=0,
$$

which confirms the weak continuity of the sum function of the RFS series (9) and Theorem 2 is proved.

Imposition of stronger condition on weights $a_{n}$ improves the result in the sense that the sum function is differentiable in probability (see Definition $\mathrm{D}_{4}$ in $\S 2$ ) which we prove in our next theorem.

TheOREM 3. Let $X(t, w), A_{n}(w)$ and $a_{n}$ have the same meaning as in Theorem 2. Then the sum function of the RFS series (9) is differentiable in probability if $a_{n}$ satisfies the condition

$$
\sum_{-\infty}^{\infty}\left|n a_{n}\right|^{2}<\infty .
$$

REMARK. Theorem 3 is an improvement over the results of [6]. Under the condition $\sum_{-\infty}^{\infty}\left|n a_{n}\right|^{2}<\infty$ they have shown the sum function to be strongly continuous in probability whereas we have shown it to be differentiable in probability.

Proof of Theorem 3. Since

$$
\sum_{-\infty}^{\infty}\left|n a_{n}\right|^{2}<\infty
$$

by the Riesz-Fischer theorem there exists a function $g \in L^{2}$ such that

$$
n a_{n}=\int_{0}^{1} e^{-2 \pi n i t} g(t) d t
$$

Let

$$
S(y, w)=\sum_{-\infty}^{\infty} a_{n} A_{n}(w) e^{2 \pi n i y} .
$$

Then 


$$
\begin{aligned}
\frac{S(y+h, w)-S(y, w)}{h} & =\sum_{-\infty}^{\infty} a_{n} A_{n}(w)\left(\frac{e^{2 \pi n i(y+h)}-e^{2 \pi n i y}}{h}\right) \\
& =\sum_{-\infty}^{\infty} a_{n} A_{n}(w) e^{2 \pi n i y}\left(\frac{e^{2 \pi n i h}-1}{h}\right) \\
& =\sum_{-\infty}^{\infty} 2 \pi n i a_{n} A_{n}(w) e^{2 \pi n i y}\left(\frac{e^{2 \pi n i h}-1}{2 \pi n i h}\right) .
\end{aligned}
$$

Denoting

$$
d_{n}=b_{n}\left(\frac{e^{2 \pi n i h}-1}{2 \pi n i h}\right)
$$

where $b_{n}=n a_{n}$, we get

$$
\frac{S(y+h, w)-S(y, w)}{h}=2 \pi i \sum_{-\infty}^{\infty} d_{n} A_{n}(w) e^{2 \pi n i y},
$$

which is a RFS series with weights $d_{n}$. Again

$$
d_{n}=b_{n}\left(\frac{e^{2 \pi n i h}-1}{2 \pi n i h}\right)=b_{n} \frac{1}{h} \int_{-h}^{0} e^{-2 \pi n i t} d t=\int_{0}^{1} \frac{1}{h} \int_{-h}^{0} g(y-t) d t e^{-2 \pi n i y} d y
$$

Thus $d_{n}$ is the Fourier coefficient of an integral which is absolutely continuous and hence belongs to $L^{p}, p>0$. So by Theorem 2

$$
\sum_{-\infty}^{\infty} d_{n} A_{n}(w) e^{2 \pi n i y}
$$

converges in probability to

$$
\int_{0}^{1} \frac{1}{h} \int_{-h}^{0} g(y-t-u) d u d X(t, w) \text {. }
$$

Thus

$$
\begin{aligned}
P\left(\left|\frac{S(y+h, w)-S(y, w)}{h}-\int_{0}^{1} g(y-t) d X(t, w)\right| \geqq \delta\right) \\
=P\left(\mid \frac{S(y+h, w)-S(y, w)}{h}-\int_{0}^{1} \frac{1}{h} \int_{-h}^{0} g(y-t-u) d u d X(t, w)\right. \\
\left.\quad+\int_{0}^{1} \frac{1}{h} \int_{-h}^{0} g(y-t-u) d u d X(t, w)-\int_{0}^{1} g(y-t) d X(t, w) \mid \geqq \delta\right) \\
=P\left(\left|\int_{0}^{1} \frac{1}{h} \int_{-h}^{0} g(y-t-u) d u d X(t, w)-\int_{0}^{1} g(y-t) d X(t, w)\right| \geqq \delta\right) \\
=P\left(\left|\int_{0}^{1}\left(\frac{1}{h} \int_{-h}^{0} g(y-t-u) d u-g(y-t)\right) d X(t, w)\right| \geqq \delta\right)
\end{aligned}
$$


$\leqq \frac{C \cdot 2^{\alpha+1}}{(\alpha+1) \delta^{\prime \alpha}} \int_{0}^{1}\left|\frac{1}{h} \int_{-h}^{0} g(y-t-u) d u-g(y-t)\right|^{\alpha} d t \quad$ (by Lemma 2)

$=K_{\alpha} \int_{0}^{1}\left|\frac{1}{h} \int_{1}^{0} g(y-t+h v)(-h d v)-g(y-t)\right|^{\alpha} d t \quad$ (taking $v=-\frac{1}{h} u$

and $K_{\alpha}$ a constant depending on $\alpha$ and $\delta^{\prime}$ )

$=K_{\alpha} \int_{0}^{1}\left|\int_{0}^{1} g(y-t+h v) d v-g(y-t)\right|^{\alpha} d t$

$=K_{\alpha} \int_{y}^{y-1}\left|\int_{0}^{1} g(x+h u) d u-g(x)\right|^{\alpha}(-d x) \quad$ (taking $\left.y-t=x\right)$

$=K_{\alpha}\left(-\int_{0}^{-1}\left|\int_{0}^{1} g(x+h u) d u-g(x)\right|^{\alpha} d x\right) \quad$ (by periodicity)

$=K_{\alpha} \int_{-1}^{0}\left|\int_{0}^{1} g(x+h u) d u-g(x)\right|^{\alpha} d x$

$=K_{\alpha} \int_{0}^{1}\left|\int_{0}^{1} g\left(x^{\prime}-1+h u\right) d u-g\left(x^{\prime}-1\right)\right|^{\alpha} d x^{\prime} \quad$ (taking $\left.x^{\prime}=x+1\right)$

$=K_{\alpha} \int_{0}^{1}\left|\int_{0}^{1} g\left(x^{\prime}+h u\right) d u-g\left(x^{\prime}\right)\right|^{\alpha} d x^{\prime}$ (by periodicity)

$=K_{\alpha} \int_{0}^{1}\left|\int_{0}^{1} g\left(x^{\prime}+h u\right) d u-\int_{0}^{1} g\left(x^{\prime}\right) d u\right|^{\alpha} d x^{\prime}$

$=K_{\alpha} \int_{0}^{1}\left|\int_{0}^{1}\left(g\left(x^{\prime}+h u\right)-g\left(x^{\prime}\right)\right) d u\right|^{\alpha} d x^{\prime}$

$\leqq K_{\alpha} \int_{0}^{1}\left(\int_{0}^{1}\left|g\left(x^{\prime}+h u\right)-g\left(x^{\prime}\right)\right| d u\right)^{\alpha} d x^{\prime}$

$\leqq K_{\alpha} \int_{0}^{1}\left[\left(\int_{0}^{1}\left|g\left(x^{\prime}+h u\right)-g\left(x^{\prime}\right)\right|^{\alpha} d u\right)^{1 / \alpha}\left(\int_{0}^{1} 1 d u\right)^{1-1 / \alpha}\right]^{\alpha} d x^{\prime}$

(by Holder's inequality)

$=K_{\alpha} \int_{0}^{1}\left(\int_{0}^{1}\left|g\left(x^{\prime}+h u\right)-g\left(x^{\prime}\right)\right|^{\alpha} d u\right)^{\alpha \cdot 1 / \alpha} d x^{\prime}$

$=K_{\alpha} \int_{0}^{1} \int_{0}^{1}|g(x+h u)-g(x)|^{\alpha} d u d x$.

Since $g \in L^{2}$ implies $g \in L^{p}, p \leqq 2$, by (12) we conclude that

$$
\lim _{h \rightarrow 0} \int_{0}^{1}|g(x+h u)-g(x)|^{\alpha} d x=0
$$

and get

$$
\lim _{h \rightarrow 0} P\left(\left|\frac{S(y+h, w)-S(y, w)}{h}-\int_{0}^{1} g(y-t) d X(t, w)\right| \geqq \delta\right)=0,
$$

which confirms that the RFS series (9) is differentiable in probablility. 
In the next theorem we consider the case $\alpha=2$ where the situation is considerably improved in the sense that the convergence of the RFS series (9) is an almost sure convergence as well as the sum function can be made almost surely continuous under some condition on the weights $a_{n}$.

THEOREM 4. Let $X(t, w)$ be a Wiener process with period one, $X(0, w)=0$ and $f \in L^{2}[0,1], A_{n}(w)$ and $a_{n}$ having the same meaning as in Theorem 2. Then we have:

(a) the RFS series (9) converges to the stochastic integral (10) almost surely and the sum function is continuous in quadratic mean.

(b) If further $a_{n}$ satisfies

$$
\sum_{-\infty}^{\infty}\left|a_{n}\right|<\infty,
$$

then the sum function is almost surely continuous.

REMarK. Theorem 4 is an improvement over [6] in two ways. The condition of continuity in quadratic mean is stronger than the continuity in probability. Besides, they have shown the almost sure convergence of the RFS series under a stronger condition $\sum_{-\infty}^{\infty}\left|n a_{n}\right|^{2}<\infty$ but we have shown it under a weaker condition.

Proof of Theorem 4. As in Theorem 2 , denoting $S_{n}(y, w)$ and $f_{n}(t)$ by

$$
S_{n}(y, w)=\sum_{-n}^{n} a_{k} A_{k}(w) e^{2 \pi k i y} \quad \text { and } \quad f_{n}(t)=\sum_{-n}^{n} a_{k} e^{2 \pi k i t}
$$

we get

$$
S_{n}(y, w)=\int_{0}^{1} f_{n}(y-t) d X(t, w) .
$$

We know (cf. Lukacs [4, p. 147]) for $g \in L^{2}[0,1]$ and a Wiener process $X(t, w)$

$$
E\left|\int_{0}^{1} g(t) d X(t, w)\right|^{2}=\sigma^{2} \int_{0}^{1}|g(t)|^{2} d t
$$

where $\sigma$ is a constant associated with the normal law of the increment of the process $X(t, w)$. Hence we get

$$
E\left|S_{n}(y, w)-\int_{0}^{1} f(y-t) d X(t, w)\right|^{2}=\sigma^{2} \int_{0}^{1}\left|f_{n}(y-t)-f(y-t)\right|^{2} d t .
$$

But by (11)

$$
\lim _{n \rightarrow \infty} \int_{0}^{1}\left|f_{n}(y-t)-f(y-t)\right|^{2} d t=0 .
$$

So 


$$
\lim _{n \rightarrow \infty} E\left|S_{n}(y, w)-\int_{0}^{1}(y-t) d X(t, w)\right|^{2} d t=0 ;
$$

which proves the convergence of the RFS series to the stochastic integral $\int_{0}^{1} f(y-t) d X(t, w)$ in quadratic mean. Now we will improve it to an almost sure convegence.

We know (cf. Lukacs [4, p. 148]) the stochastic integral $\int_{a}^{b} g(t) d X(t, w)$ for continuous $g(t)$ in $[a, b]$ and a Wiener process $X(t, w)$ is a random variable with normal distribution having mean zero and finite variance $\int_{a}^{b}|g(t)|^{2} d t$. So

$$
A_{n}(w)=\int_{0}^{1} e^{-2 \pi n i t} d X(t, w)
$$

is a random variable with mean zero and finite variance. Also we know (cf. Doob [2, p. 427]) for a process $Y(t, w)$ with orthogonal increments and for $g, f \in L^{2}[a, b]$

$$
E\left(\int_{a}^{b} f(t) d Y(t, w) \overline{\int_{a}^{b} g(t) d Y(t, w)}\right)=\int_{a}^{b} f(t) \overline{g(t)} d t,
$$

where $\overline{g(t)}$ denotes the complex conjugate of $g(t)$.

Now $X(t, w)$ being a Wiener process has orthogonal increments and thus we get

$$
\begin{aligned}
E A_{n}(w) \overline{A_{m}(w)} & =E \int_{0}^{1} e^{-2 \pi n i t} d X(t, w) \overline{\int_{0}^{1} e^{-2 \pi m i t} d X(t, w)} \\
& =\int_{0}^{1} e^{-2 \pi i t(n-m)} d t=0, \quad(m \neq n)
\end{aligned}
$$

Thus $A_{n}(w)$ is a sequence of independent random variable because of its normality. Further, we notice

$$
\sum_{-\infty}^{\infty} E\left|a_{n} A_{n}(w) e^{2 \pi n i y}\right|^{2}=\sum_{-\infty}^{\infty} E\left|a_{n} \int_{0}^{1} e^{2 \pi n i(y-t)} d X(t, w)\right|^{2}=\sum_{-\infty}^{\infty} \int_{0}^{1}\left|a_{n}\right|^{2} d t \quad(\text { by (14)). }
$$

Since

$$
f \in L^{2}[0,1] \text { implies } \sum_{-\infty}^{\infty}\left|a_{n}\right|^{2}<\infty \text {, }
$$

we get

$$
\sum_{-\infty}^{\infty} E\left|a_{n} A_{n}(w) e^{2 \pi n i y}\right|^{2}<\infty
$$

Thus $S_{n}(y, w)$ constitutes a sum of normally distributed, independent random variables where the sum of variance is finite. Hence by the Kolmogorov theorem on sums of independent random variable, $S_{n}(y, w)$ converges almost surely to the stochastic integral 


$$
\int_{0}^{1} f(y-t) d X(t, w)
$$

To show the continuity property of the sum function we see that by (14)

$$
E\left|\int_{0}^{1} f(x-t) d X(t, w)-\int_{0}^{1} f(y-t) d X(t, w)\right|^{2}=\int_{0}^{1}|f(x-t)-f(y-t)|^{2} d t
$$

Now by (12) one easily see that the integral on the right tends to zero as $x \rightarrow y$. Thus the sum function is continuous in quadratic mean.

For the second part of the theorem we notice that by Weistrass $M$-test the series (9) converges uniformly for almost all $y$ to a continuous function almost surely if

$$
\sum_{-\infty}^{\infty}\left|a_{n} A_{n}(w)\right|<\infty \quad \text { almost surely . }
$$

For this it is sufficient to show that

$$
\sum_{-\infty}^{\infty} E\left|a_{n} A_{n}(w)\right|<\infty \text {. }
$$

Now the condition $\sum_{-\infty}^{\infty}\left|a_{n}\right|<\infty$ in Theorem 4 ensures the statement (15) since $\left|A_{n}(w)\right|$ is a bounded sequence and thus the second part of Theorem 4 is completed.

Our next theorem deals with the case $\alpha=1$ where we show that the series $(9)$ is convergent in $(C, 1)$ probability to the stochastic integral (10).

THEOREM 5. Let $X(t, w)$ be a stable process of index $\alpha, \alpha=1$, with period one, while $f \in L^{1}[0,1], A_{n}(w)$ and $a_{n}$ have the same meaning as in Theorem 2. Then the series (9) is convergent in $(\mathrm{C}, 1)$ probability to the stochastic integral (10).

REMARK. Combining Theorems 2, 4 and 5 we see that the RFS series (9)

(a) converges almost surely for $\alpha=2$

(b) converges in probability for $1<\alpha<2$

(c) converges in (C, 1) probability for $\alpha=1$.

Since $(a) \Rightarrow(b)$, it may appear that whenever the index of the process decreases, the result obtained becomes weaker. Now it follows from (cf. Maddox [5]) that

$$
\begin{aligned}
& S_{n} \rightarrow s \text { in probability } \\
& \Rightarrow \sigma_{n} \rightarrow s \text { in probability }\left(\sigma_{n} \text { denotes } \frac{S_{0}+S_{1}+\cdots+S_{n-1}}{n}\right)
\end{aligned}
$$

provided $\left|S_{n}\right| \leqq g$ for some $g \in L^{1}$. 
Hence in our case if we can show that

$$
\left|S_{n}\right| \leqq g \text { for some } g \in L^{1}
$$

then $(b) \Rightarrow(c)$. In particular, if the Fourier coefficient $a_{n}$ is such that $A=\sum_{-\infty}^{\infty}\left|a_{n}\right|<\infty$ then $\left|S_{n}\right| \leqq A \int_{0}^{1} d X(t, w)$ and $(\mathrm{a}) \Rightarrow(\mathrm{b}) \Rightarrow$ (c) follows.

Proof OF TheORem 5. Let

and

$$
\sigma_{n}^{\prime}(y, w)=\frac{S_{0}+S_{1}+\cdots+S_{n-1}}{n}
$$

$$
\sigma_{n}(t)=\frac{f_{0}+f_{1}+\cdots+f_{n-1}}{n},
$$

where $S_{n}(y, w)$ and $f_{n}(t)$ have the same meaning as in Theorem 2. We see as before

Also we get

$$
S_{n}(y, w)=\int_{0}^{1} f_{n}(y-t) d X(t, w) .
$$

Hence

$$
\sigma_{n}^{\prime}(y, w)=\int_{0}^{1} \sigma_{n}(y-t) d X(t, w) .
$$

$$
\sigma_{n}^{\prime}(y, w)-\sigma_{m}^{\prime}(y, w)=\int_{0}^{1}\left(\sigma_{n}(y-t)-\sigma_{m}(y-t)\right) d X(t, w) .
$$

Considering $\alpha=1$ in Lemma 2 we get

$$
P\left(\left|\sigma_{n}^{\prime}(y, w)-\sigma_{m}^{\prime}(y, w)\right|>\delta\right) \leqq \frac{2 C}{\delta} \int_{0}^{1}\left|\sigma_{n}(y-t)-\sigma_{m}(y-t)\right| d t .
$$

But we know (cf. Zygmund [9, p. 144]):

(a) The necessary and sufficient condition for $\sum S_{n}(x)$ to belong to $L^{1}$ is that

(b)

$$
\lim _{m, n \rightarrow \infty} \int_{0}^{1}\left|\sigma_{m}-\sigma_{n}\right|=0
$$

$$
\lim _{n \rightarrow \infty} \int_{0}^{1}\left|\sigma_{n}-f\right|=0,
$$

where $S_{n}$ and $\sigma_{n}$ denote the $n$th partial sum and $n$th Cessàro sum of the Fourier series of $f$, respectively.

Thus $f \in L^{1}[0,1]$ implies

$$
\lim _{m, n \rightarrow \infty} \int_{0}^{1}\left|\sigma_{n}(y-t)-\sigma_{m}(y-t)\right| d t=0,
$$

which confirms the convergence of $\sigma_{n}^{\prime}$ in probability. Again we see 


$$
\begin{aligned}
P\left(\mid \sigma_{n}^{\prime}(y, w)\right. & \left.-\int_{0}^{1} f(y-t) d X(t, w) \mid>\delta\right) \\
= & P\left(\left|\int_{0}^{1}\left(\sigma_{n}(y-t)-f(y-t)\right) d X(t, w)\right|>\delta\right) \\
& \leqq \frac{2 C}{\delta} \int_{0}^{1}\left(\sigma_{n}(y-t)-f(y-t)\right) d t \quad(\text { by Lemma 2) }
\end{aligned}
$$

$\sigma_{n}(y-t)$ being the Cessàro $n$th partial sum of the Fourier series of $f$ which is in $L^{1}$, we get

$$
\lim _{n \rightarrow \infty} \int_{0}^{1}\left|\sigma_{n}(y-t)-f(y-t)\right| d t=0
$$

which concludes the convergence of the series $(9)$ in $(C, 1)$ probability to the stochastic integral (10).

If we consider the weights $a_{n}$ to be the Fourier coefficients of a weaker class of functions $f$ which are not integrable but $|f| \log ^{+}|f|$ is integrable, then the RFS series (9) will converge to the stochastic integral (10) in probability as we show in the corollary below.

CoROLlary 1. If $X(t, w)$ is a symmetric stable process of index $\alpha$, $\alpha=1$, and $a_{n}$ are the Fourier coefficients of $f$ such that $|f| \log ^{+}|f|$ is integrable, then the series (9) converges in probability to the stochastic integral (10).

For the proof of the corollary we require the following lemma.

Lemma 3 (cf. Zygmund [9, p. 107]). If $|f| \log ^{+}|f|$ is integrable, then

$$
\lim _{n \rightarrow \infty} \frac{1}{2 \pi} \int_{0}^{2 \pi}\left|f-S_{n}\right| d t=0
$$

where $S_{n}$ denotes the $n$-th partial sum of the Fourier series of $f$.

Proof of Corollary 1. First we notice that the existence of the stochastic integral $\int_{a}^{b} f(t) d X(t, w)$, when $|f| \log ^{+}|f|$ is integrable, can be shown exactly as in Theorem 1 by Lemma 3 . Then proceeding exactly as in Theorem 2 and using Lemma 3 instead of statement (11) we get the required result.

In the next theorem we discuss the case $0<\alpha<1$. When $a_{n}$ is the Fourier coefficient of an $L^{p}$ function for $0<p<1$, the RFS series (9) converges in probability to the stochastic integral (10) only under a different set of conditions on weights $a_{n}$.

THEOREM 6. If $X(t, w)$ is a symmetric stable process of index $\alpha$, $0<\alpha<1$, and $a_{n}$ is the Fourier coefficient of an $L^{p}$ function, $0<p<1$, 
then the series (9) converges in probability to the stochastic integral (10) provided

$$
\lim _{n \rightarrow \infty} a_{n}=0 \quad \text { and } \quad \sum_{-\infty}^{\infty}\left|a_{n+1}-a_{n}\right|<\infty .
$$

For the proof of the theorem we require the following lemma.

Lemma 4 (cf. Bary [1, p. 215]). If $a_{n}$ is the Fourier coefficient of $f \in L^{p}, 0<p<1$, with $\lim _{n \rightarrow \infty} a_{n}=0$ and $\sum_{-\infty}^{\infty}\left|a_{n+1}-a_{n}\right|<\infty$, then

$$
\lim _{n \rightarrow \infty} \int_{0}^{1}\left|f-S_{n}\right|^{p} d X=0 \text {, }
$$

where $S_{n}$ is the n-th partial sum of the Fourier series of $f$.

Proof of Theorem 6. Proceeding as in Theorem 1 and using Lemma 4 instead of statement (11) the existence of the stochastic integral $\int_{a}^{b} f(t) d X(t, w)$ for $f \in L^{p}, 0<p<1$, and $X(t, w)$ a stable process of index $\alpha, 0<\alpha<1$, can be shown in the sense of convergence in probability. Then the proof follows the pattern of the proof of Theorem 2 .

\section{REFERENCES}

[1] N. K. Bary, A Treaties on Trigonometric Series, Vol. II, Pergamn Press, Oxford, London, Edinburg, New York, Paris, Frankfurt, 1964.

[2] J. L. Dooв, Stochastic Processes, John Wiley \& Sons, New York, Brisbane, Toronto, 1953.

[3] T. Kawata, Fourier Analysis in Probability Theory, Academic Press, New York and London, 1972.

[4] E. Lukacs, Stochastic Convergence, Academic Press, New York, San Francisco, London, 1975.

[5] I. J. MADDOX, Toeplitz transformation and convergence in measure, J. London Math. Soc., 41, 1966, 733-736.

[6] M. N. Mishra, N. N. Nayak and S. Pattanayak, Some continuity properties of a random Fourier-Stieltjes series, J. of Mathematical and Physical Sciences, 14, 1980, 259-272.

[7] G. Samal, Fourier series associated with sample function of stochastic processes, Proc. Camb. Phil. Soc. 67, 1970, 101-106.

[8] G. Samal and M. N. Mishra, Continuity property of a random Fourier-Stieltjes series, Bulletin Calcutta Math. Soc. 63, 1971, 97-100.

[9] A. Zygmund, Trigonometric Series, Cambridge Univ. Press, London, New York, Mel. bourne, 1977.

School of Mathematical Sciences

SAMBALPUR UNIVERSITY, ORISSA

INDIA 
